\title{
DEVELOPMENT OF PRESTRIATE VISUAL PROJECTIONS IN THE MONKEY AND HUMAN FETAL CEREBRUM REVEALED BY TRANSIENT CHOLINESTERASE STAINING ${ }^{1}$
}

\author{
IVICA KOSTOVIC* AND PASKO RAKIC $\ddagger^{2}$ \\ * Department of Anatomy, University of Zagreb, Yugoslavia, and $\$$ Section of Neuroanatomy, Yale University Medical School, \\ New Haven, Connecticut 06510
}

Received February 22, 1983; Revised May 19, 1983; Accepted June 29, 1983

\begin{abstract}
Cholinesterase (ChE) staining was used to reveal the timing and pattern of development of afferents to the prestriate visual cortex (areas 18,19,20, and 21 of Brodmann) in a series of developing human and monkey fetal brains. This investigation was possible because the nucleus pulvinaris of the thalamus, the main source of subcortical projections to the prestriate cortex, displays positive reactivity after thiocholine incubation during the last three quarters of gestation, while neighboring thalamic nuclei that project to the adjacent neocortical areas are unstained. Staining of the pulvinar and its prestriate projections passes through six broad stages. Stage I begins in both species at the end of the first third of gestation. Positively stained fibers originate from the pulvinar and enter but do not extend beyond the hemispheric stalk. During stage II, pulvinar axons gradually invade the intermediate zone of the occipital lobe, and in stage III they reach the level of the subplate zone. In stage IV, which occurs around mid-gestation in both species, cholinesterase-positive fibers accumulate within the subplate zone subjacent to the developing prestriate cortex. During stage V, ChE-positive fibers penetrate the prospective prestriate cortex but do not yet form the alternating columnar pattern characteristic of pulvinar input to this area in the adults. Rather, ChE activity is concentrated in two continuous bands situated within prospective layers III-IV and VI; also a narrow band is visible in upper layer I. In stage V a clear histochemical border forms between prestriate and striate areas with ChE activity in prospective area 17 limited mostly to the superficial strata of layers I and II. This histochemical differentiation precedes the emergence of cytoarchitectonic landmarks. During stage VI, which begins in the last fifth of gestation in both species, the pulvinar become progressively less stainable and its projections can no longer be traced by ChE histochemistry.
\end{abstract}

In the adult primate, prestriate visual cortex is well differentiated from primary visual cortex by its cytoarchitectonic characteristics as well as by the source and distribution of its thalamic afferents. In the rhesus monkey, as in other mammals, the prestriate cortex (areas $18,19,20$, and 21 of Brodmann) receives its thalamic innervation primarily from the pulvinar nucleus of the thalamus (Cragg, 1969; Zeki, 1969a, b; Benevento and Rezak, 1976; Curcio and Harting, 1978). Although a small

\footnotetext{
${ }^{1}$ This research was supported in part by United States Public Health Service Grant EY 02593, by United States PI -480 Foreign Currency Program 02-006, and by United States-Yugloslav Joint Board Funds 02-081-N.

${ }^{2}$ To whom correspondence should be addressed, at Section of Neuroanatomy, Yale University Medical School, 333 Cedar Street, New Haven, CT 06510.
}

contingent of axons from the pulvinar terminates in the primary visual cortex (area 17), they are sparse and distributed differently from geniculocortical fibers that are the main source of innervation of this cortical area.

No information is available on the development of the prestriate cortex and its thalamic innervation. The lack of suitable anatomical methods for analysis of connectivity in fetal brain has been a major obstacle to the study of early development of cerebral cortex. Only recently, transneuronal transport of radioactive amino acids from the eye has aided in the analysis of developing geniculocortical projections (Rakic, 1976a, 1983). However, no equally suitable method has been available for detecting projections of nonprimary relay thalamic nuclei such as the pulvinar. Recently, it has become clear that various subdivisions of the developing primate thalamus including the pulvinar nucleus exhibit different susceptibility 
and/or schedules of emergence of cholinesterasic activity (Kostovic and Goldman-Rakic, 1983). Enzymes that are in the adult brain are involved in degradation of acetylcholine and are present also in noncholinergic systems during embryogenesis and have long been recognized to play a role in developmental processes (e.g., Csillik et al., 1964; Filogamo and Marchisio, 1965; Krnjevic and Silver, $1965,1966)$. In the present study we used Lewis's modification of the acetylthiocholine iodide method of KoelleFreidenwald (Krnjevic and Silver, 1965, 1966) to reveal transient cholinesterase activity and thus to study the development of the pulvinar and its outgrowing projections to the cerebral cortex.

\section{Materials and Methods}

The material consisted of 14 human and 16 monkey brains of various pre- and postnatal ages that were processed with several modifications of acetylcholinesterase (AChE) histochemistry. In addition, Nissl-stained sections from specimens of corresponding ages were used to determine the cytoarchitectonic boundaries and levels of cytodifferentiation of pulvinar and visual cortical areas at corresponding developmental stages.

The brains from eight human fetuses of 10.5, 15, 18, $22,23,26,28$, and 35 weeks (W) of gestation were processed for AChE reactivity. The gestational age of each fetus (expressed in weeks (W)) was estimated on the basis of crown-rump length and/or pregnancy records and was expressed as ovulation age (Olivier and Pineau, 1961). In addition we studied one newborn, five infants aged $1,3,5.5,8$, and 18 months, and two adults. Tissue blocks used for AChE staining were fixed by immersion in $10 \%$ formalin/saline for periods of up to 4 days. The blocks were sectioned on a freezing microtome at $80 \mu \mathrm{m}$. All blocks were cut in the coronal plane except for two which were sectioned horizontally and one which was cut sagittally.

The brains from 11 rhesus monkey fetuses, 1 newborn, 2 infants aged 2 and 12 months, respectively, and 2 adult monkeys were processed for AChE histochemistry. The ages of the monkey fetuses (in embryonic days (E)) were determined from breeding records and are accurate to \pm 2 days. They are as follows: E47, E54, E60, E71, E83, E93, E102, E117, E120, E123, and E155. All monkey brains were fixed by intracardial perfusion with quarterstrength Karnovsky fixative as described previously (Rakic, 1972) and postfixed in formalin/saline and su- crose/formalin for 1 to 4 days before being sectioned coronally or sagittally at $50 \mu \mathrm{m}$.

Most sections of the human and monkey brains were incubated by using a modification of the acetylthiocholine iodide method (Krnjevic and Silver, 1965, 1966). Histochemical specificity was tested by treatment of adjacent sections in incubation medium containing $10^{-4}$ $\mathrm{M}$ eserine to inhibit both butyrylcholinesterase (BuChE) and AChE, or $10^{-4} \mathrm{M}$ ethopropazine hydrochloride and $10^{-7} \mathrm{M}$ Haloxone to inhibit BuChE. Also sections from cases at early, middle, and late developmental stages were preincubated in iso-OMPA $\left(10^{-3}\right.$ and $\left.10^{-4} \mathrm{M}\right)$ for more specific inhibition of pseudocholinesterase (Silver, 1974). Reaction product was developed with sodium sulfide in $0.2 \mathrm{M}$ acetic acid after incubation times of up to $48 \mathrm{hr}$. Some tissue was processed by the "direct coloring" method of Karnovsky and Roots (1964) with the incubation medium and inhibitors suggested by Broderson et al. (1974). In selected sections, staining was intensified with 0.1 to $1.0 \%$ silver nitrate (Geneser-Jensen and Blackstad, 1971). The direct coioring method stained the thalamic region well hut proved to be less sensitive for staining of developing axons at the middle gestational ages when they are entering the telencephalon and begin to invade the cortical plate.

In both human and monkey fetuses, pulvinar neurons display strong cholinesterasic (ChE) activity during the last two-thirds of gestation. Because staining at these critical developmental ages may be due to the presence of various molecular forms of $\mathrm{ChE}$ which are difficult to assess with histochemical methods (see Kostovic and Goldman-Rakic, 1984, and "Discussion"), reactivity in this study is simply referred to as ChE reactivity.

\section{Results}

The development of prestriate projections can be divided, somewhat arbitrarily, into six developmental stages hased on $\mathrm{ChF}$ staining intensity and the spread of activity via pulvinar axons to their eventual targets in the cortical plate (Table I). Each of these morphologically definable stages may represent a distinct step in the genesis of these afferent connections. In addition, delineation of these stages allows comparison of corresponding developmental events in humans and monkeys.

Stage I. During this stage, which occurs between W11 and W13 in humans and around E47 in monkeys, the anlage of the pulvinar nucleus can be recognized in Nissl-

TABLE I

ChE reactivity in developing human and monkey pulvinar neurons ${ }^{\alpha}$

\begin{tabular}{|c|c|c|c|c|c|c|c|c|}
\hline \multirow{2}{*}{ Stage $^{b}$} & \multirow{2}{*}{ Human } & \multirow{2}{*}{ Monkey } & \multirow{2}{*}{ Pulvinar Neurons } & \multicolumn{2}{|c|}{ Fibers in IZ } & \multicolumn{2}{|c|}{ Subplate } & \multirow{2}{*}{ Cortical Plat } \\
\hline & & & & Present & Spread & Present & Border & \\
\hline I & W11-13 & $\mathrm{E} 47$ & + & $+/-$ & - & - & - & - \\
\hline II & W14-16 & E54-59 & ++ & + & $+1-$ & - & - & - \\
\hline III & W17-20 & E60-79 & +++ & ++ & ++ & $+/-$ & - & - \\
\hline IV & W21-25 & E80-99 & +++ & +++ & +++ & +++ & ++ & + \\
\hline $\mathrm{V}$ & W26-34 & E100-124 & ++ & ++ & +++ & ++ & +++ & +++ \\
\hline $\mathrm{VI}$ & W35 & F125- & $+/-$ & - & - & - & - & - \\
\hline
\end{tabular}

a The reactivity of $\mathrm{ChE}$ staining was subjectively graded as follows: - , absent; $+/-$, sparse; + , moderate;,++ dense;,+++ peak.

${ }^{b}$ Delineation of stages is somewhat arbitrary due to the limited number of fetal specimens in both species and estimation of ages in fetal weeks (W) for human and embryonic (E) days for monkey (see "Materials and Methods"). 
stained sections in the posterior moiety of the developing thalamus in both humans (Yakovlev, 1969; Rakic, 1974a) and monkeys (Ogren and Rakic, 1981). Although precise cytoarchitectonic borders of the pulvinar are still not sharply delineated, several well defined landmarks such as the habenular nucleus, the fasciculus retroflexus, the ventral and dorsal lateral geniculate nuclei, and the faint but definitely visible centrum medianum all aid in locating pulvinar anlage (Fig. 1A). This first developmental stage is characterized by a moderate but clearly positive ChE reactivity in the pulvinar region of the thalamus. The reactivity is somewhat enhanced by longer incubation times (Fig. $1 B$ ) which, in addition, expose bundles of fibers that emerge from the pulvinar and pass via the inferior and posterior thalamic radiations into the occipital lobe (Fig. 1C). However, the stained fibers do not invade the middle portion of the intermediate zone and they stop short of entering the posterior half of the occipital lobe.

At these early embryonic ages, the thalamus of humans and monkeys exhibits a remarkable specificity in $\mathrm{ChE}$ staining. The territory of the pulvinar anlage is reactive whereas the adjacent thalamic nuclei of the posterolateral thalamus are not. In coronal sections the prospective pulvinar appears as a wedge-shaped $\mathrm{ChE}$-positive area with clear borders along the dorsolateral edge and mediobasal aspect of the posterior thalamus. Both the lateral geniculate (LGd) and centrum medianum nuclei (Fig. 1B) are distinguishable cytoarchitectonically and their cells are not stained by the ChE method at any of the stages examined in this study. Although the pulvinar anlage merges rostrally with the ChE-positive mediodorsal nucleus, these two structures can be distinguished by their relative positions and the orientation of emerging fiber tracts (Kostovic and Goldman-Rakic, 1983). Likewise, the ChE-positive habenular nucleus which lies dorsomedially can be distinguished by its small round shape, its cytoarchitectonic properties, its exceptionally dense staining, and the emergence of the large habenulo-interpeduncular tract (Fig. $1, B$ and $D$ ).

Although ChE-positive fibers that originate from the pulvinar enter the territory of the temporoparieto-occipital confluence within the laterobasal convexity of the telencephalon, these axons do not invade the deeper and more posterior parts of the occipital lobe (Fig. $1 C$ ) and none are visible in the sections cut across the posterior portion of the occipital pole (not shown). The developing cortical plate at this stage is still relatively thin and, according to $\left[{ }^{3} \mathrm{H}\right]$ thymidine data analysis in the monkey, contains only neurons of the infragranular layers in both striate (Rakic, 1974b) and prestriate cortex (Rakic, 1976a). Neither area has ChE activity at this fetal stage.

Stage II. During the second stage, which corresponds to W14 to W16 in the human and about E54 to E59 in the monkey, ChE activity within pulvinar neurons increases in intensity. Although the entire territory of the pulvinar is well stained, the medial and lateral moieties begin to show a "patchy" distribution of ChE (Fig. $2 B$ ), while the inferior portion situated between and above the lateral and medial geniculate nuclei remains for the most part dense and homogeneous. At this fetal age the cytoarchitectonic boundaries of thalamic nuclei begin to emerge and can be discerned in corresponding cresyl violet-stained sections in both monkeys (Ogren and Rakic, 1981) and humans (Yakovlev, 1969; Rakic, 1974a). The lateral portion of the pulvinar begins to show a lower neuronal packing density (Fig. $2 A$ ) with neurons separated into bands by cell-free, fiber-rich fasicles. These fasicles are deeply stained by the ChE method (Fig. $2 B$ ) and can be followed as they enter the occipital lobe and become distributed throughout the intermediate zone (Fig. $2 C$ ). They form a particularly dense band within the corpus semiovale in a territory that corresponds to the inner stratum of the thalamic radiation (Fig. 2, $D$ and $E$ ).

At more caudal levels, fibers of the posterior thalamic radiation are situated close to another intensely $\mathrm{ChE}$ stained fiber bundle. This bundle emanates from the external capsule and presumably originates in the rostrobasal part of the forebrain. In stage II, ChE-positive staining still cannot be detected within the subplate zone or the developing cortical plate. At this fetal age the cortical plate has become somewhat thicker but, as at the earlier stage, does not contain neurons in superficial layers II to IV (Rakic, 1974b). The cortical plate in the primate occipital lobe is still relatively immature; its densely packed neurons have not yet been subdivided into cytoarchitectonic areas.

Stage III. This stage, which occurs between W17 and W20 in human fetuses and between E60 and E79 in monkeys, shows peak ChF activity in the pulvinar and in its cortical projections. The staining is so intensive that one cannot distinguish individual cells. The main boundaries of the pulvinar are difficult to distinguish as it appears to merge anteriorly with the lateral posterior nucleus of the thalamus. However, the ventral and lateral borders of the pulvinar can readily be distinguished from the adjacent LGd and centrum medianum (Fig. $3 A$ ). At higher power microscopy individual neurons in the pulvinar display cytological signs of a more advanced stage of differentiation in both humans (Yakovlev, 1969) and monkeys (Ogren and Rakic, 1981). Electron microscopic examination of the thalamus in human fetuses of corresponding ages has revealed a small number of synapses within the pulvinar neuropil at this time (Rakic, 1974a). As observed at the earlier stages, the lateral and medial moieties of the pulvinar have a nonhomogeneous distribution of ChE activity (Figs. $3 B$ and $4, A$ and $B$ ) while the inferior pulvinar remains uniformly and densely stained (Figs. $3 B$ and $4 B$ ). Again, the inferior pulvinar is stained deeply in contrast to the LGd and centrum medianum which remains unstained (CM and $L G d$ in Fig. $3 B$ ).

At this stage, pulvinar fibers enter the occipital lobe in large numbers (Fig. $4 A$ ). They can be followed into the optic radiation, intermediate, and subplate zone (Fig. $4 C$ ). The subplate zone at this stage is prominent in both human (Kostovic an Molliver, 1974) and monkey telencephalon (Rakic, 1976b, 1977b) and contains a relatively well defined row of immature neurons intermixed with a prominent plexus of growing fibers. Although the occipital cortex has acquired almost all of its neurons at this fetal age in both the monkey (Rakic, 1974b, 1976a) and human (Sidman and Rakic, 1973), it is still ChE negative 
(Table I). Within the subplate zone, the fibers mostly invade territories that lie directly below the prospective prestriate cortex and avoid the area in the depth of calcarine fissure or lateral occipital lobe that corresponds to the future primary visual cortex. However, the border between histochemically positive and negative areas is not sharp. Autoradiographic analysis of the occipital lobe of fetuses injected with radioactive tracers in one eye
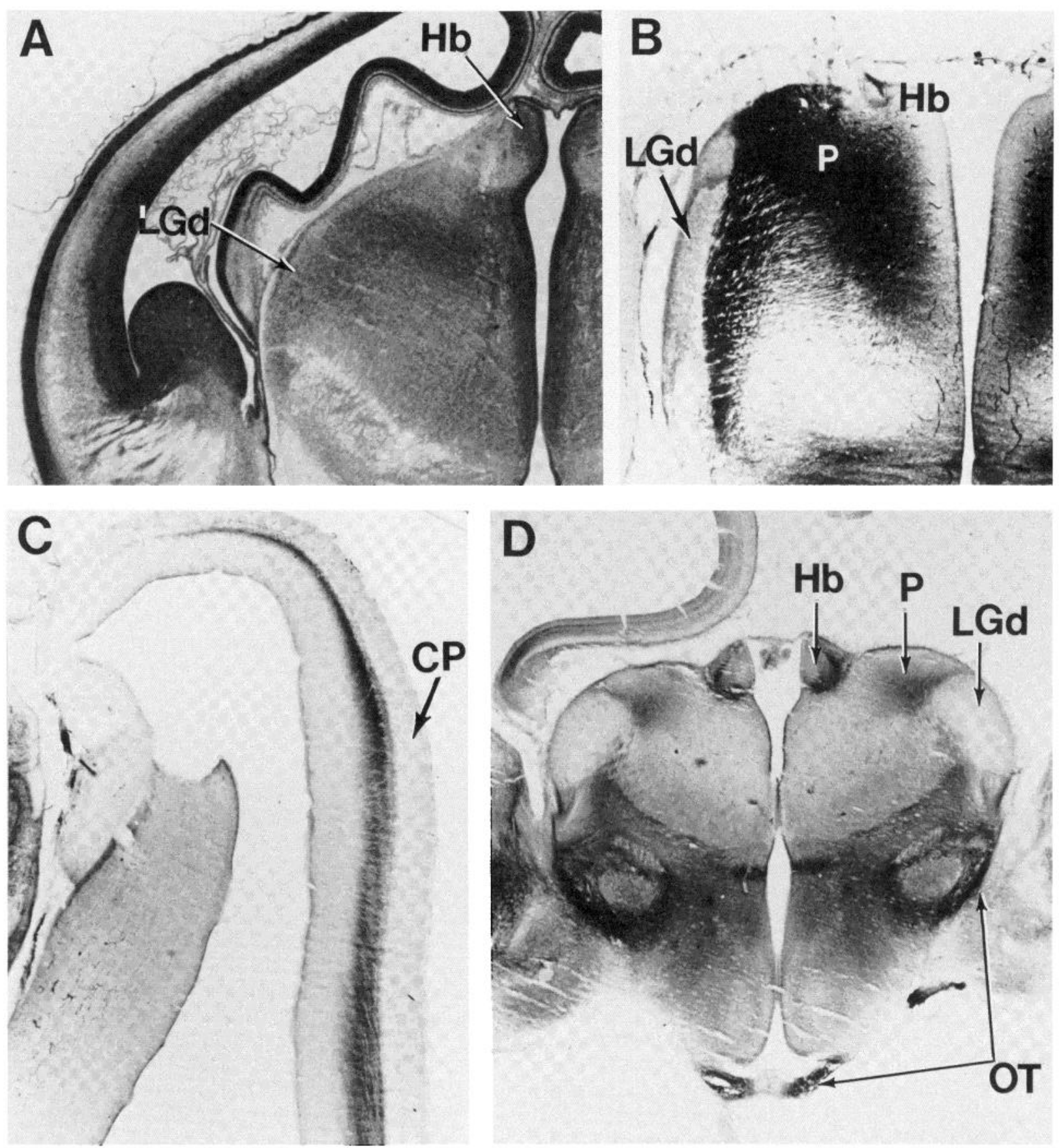

Figure 1. A, Coronal section through the posterior portion of the thalamus in a $10^{1 / 2}$-week-old human fetus stained using the cresyl violet method. The territory of the pulvinar anlage is recognized by the position of the prospective dorsal lateral geniculate nucleus $(L G d)$, which at this fetal age is situated at the lateral wall of the diencephalon, and by the habenular nucleus $(H b)$, which lies dorsally. $B$, Oblique section through the posterior portion of the thalamus in another $10^{1 / 2}$-week-old human fetus processed according to the thiocholine iodide histochemical method. Although the plane of the section is somewhat different, the unstained LGd and $\mathrm{Hb}$ are clearly distinguished from the developing pulvinar $(P)$ which is stained positively. $C$, The distribution of ChE-positive pulvinar fibers running through the occipital lobe of the same specimen that is illustrated in $B$. Note that positively stained axons are confined to the intermediate zone and do not invade the cortical plate $(C P)$ nor do they reach the occipital pole. $D$, Coronal section through the diencephalon of a 54-day-old monkey fetus stained using ChE histochemistry. Note light positive staining of the pulvinar region $(P)$ delineated laterally by the unstained dorsal lateral geniculate nucleus $(L G d)$ and mediodorsally by the highly positive habenular nucleus $(H b)$. The optic tract $(O T)$ and the basal ganglia are also well stained at this fetal age in monkeys when silver intensification is applied. 

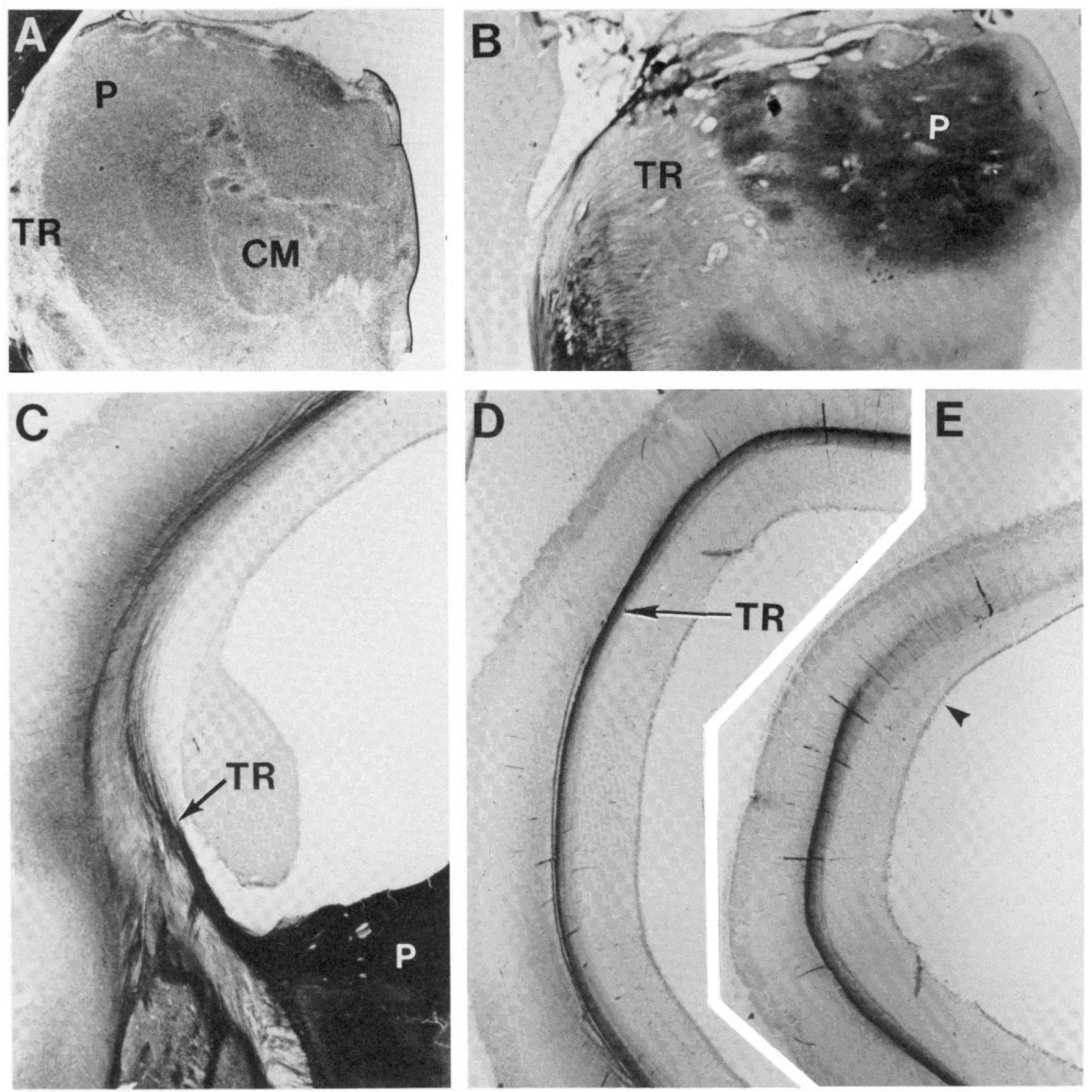

Figure 2. Appearance of the posterior region of the thalamus in the coronal section of a 15 -week-old human fetus stained by the cresyl violet method. $A$, The pulvinar region is demarcated laterally by the thalamic radiation $(T R)$ and medioventrally by the well defined centrum medianum $(C M)$. B. Posterior thalamus in another 15 -week-old human fetus displaying "patchy" positive ChE reaction in the developing pulvinar $(P)$ and well stained projections through the posterior thalamic radiation $(T R)$. $C$, Horizontal section across the pulvinar $(P)$ of a 16-week-old human fetus showing the course of the pulvinar axons forming the posterior thalamic radiation $(T R)$ entering the occipital lobe. ChE-positive axons run external to the thalamic radiation and are in continuity with the external capsule. $D$ and $E$, A montage displays the course of the positively stained pulvinar fibers ( $T R$ ) running through the intermediate zone of the occipital lobe. Note that the fibers do not invade the territory of the occipital pole (arrowhead).

shows that ChE-free regions of the subplate zone correspond to regions occupied by the geniculocortical fibers (Rakic, 1976b, 1983).

Stage IV. During this stage, which begins in human fetuses between W21 and W25 and in monkey between E80 and E99, the distribution and intensity of reaction product remain the same within the posterior thalamic region (Fig. $5 B$ ) but emerge for the first time in the interlaminar spaces within the LGd (Fig. 6A). The darkly stained pulvinar fibers enter the occipital pole and form a well defined thalamic radiation. At these fetal ages, the border between the stained and unstained portions of 

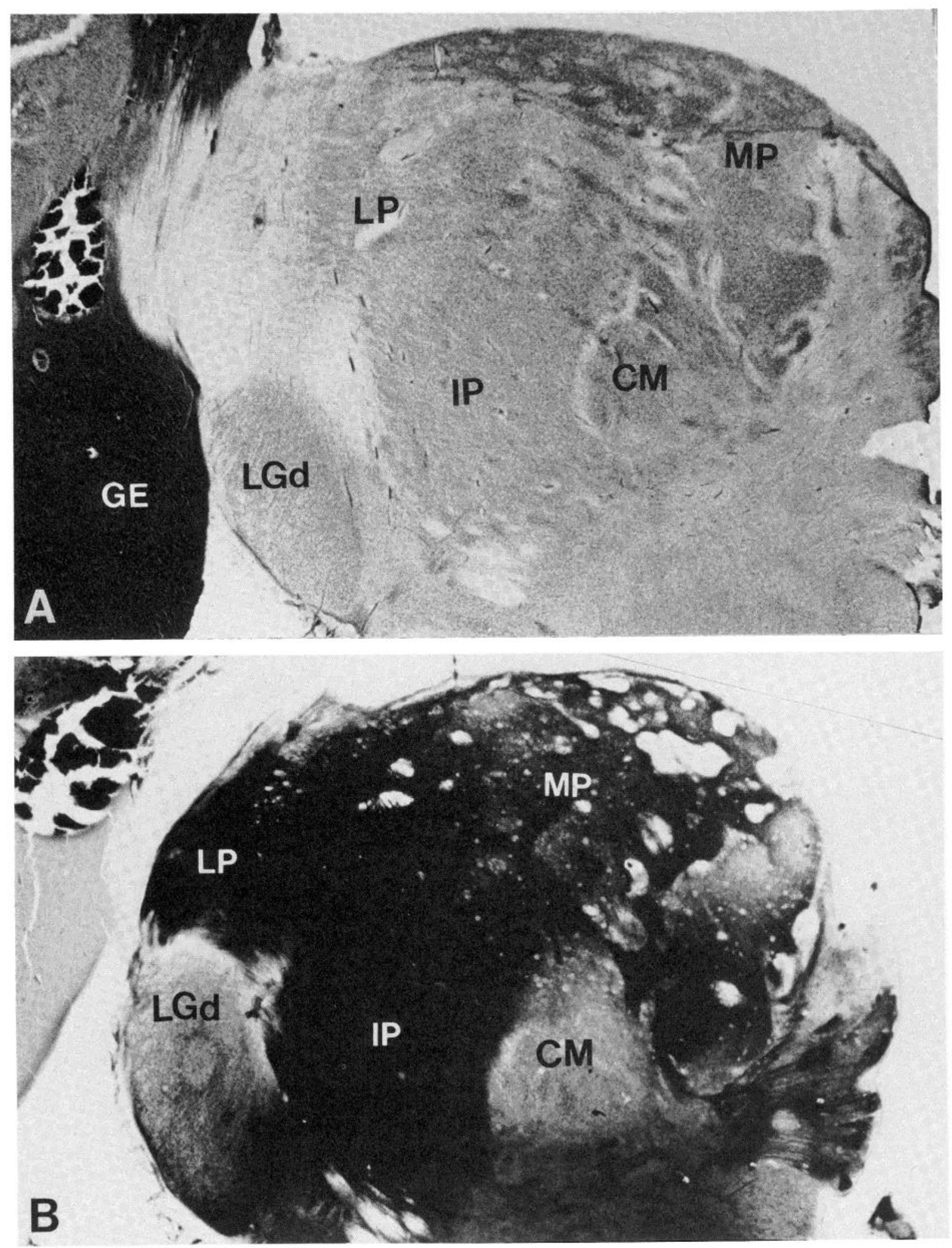

Figure 3. A, Coronal section across the posterior thalamus in an 18 -week-old human fetus stained using the cresyl violet method. By this age, the pulvinar region shows cytoarchitectonic differentiation into a medial $(M P)$, lateral $(L P)$, and inferior $(I P)$ moiety. The borders of the pulvinar complex are now easily recognized by clearly outlined landmarks of the dorsal lateral geniculate nucleus $(L G d)$ and centrum medianum $(C M)$. The ganglionic eminence $(G E)$ which produces neostriatal cells is very prominent at this age. $B$, A more posterior section across the same specimen stained using ChE histochemistry. Note intense positive reaction in the region of the inferior pulvinar $(I P)$ and "patchy" staining of the medial pulvinar $(M P)$. In contrast, the dorsal lateral geniculate nucleus $(L G d)$ and the centrum medianum $(C M)$ remain unstained. 
the subplate zone becomes sharp, and a small contingent of pulvinar fibers begins to enter the developing cortical plate (Table I, Figs. 5, $A$ and $C$, and $6 B$ ). The entrance of fibers in the developing cortex is followed by a parallel and systematic decrease in staining activity of the subplate zone. However, the fibers within the cortical plate form continuous sheets and do not exhibit the adult-like striped pattern of high and low staining intensity (Fig. $6 C$ ). In both species ChE-positive areas correspond largely to the cortex of the lateral, dorsal, and ventral portions of the occipital lobe. However, the posterior pole, a portion of the medial surface, and the depth of calcarine fissure-all parts of prospective area 17-are stain free (Figs. $5 A$ and $6 C$ ).

A novel and potentially significant observation is that the presence of $\mathrm{ChE}$ reactivity at this stage permits detection of the prospective cytoarchitectonic border between areas 17 and 18 before this border is visible in Nissl-stained material (cf. Fig. 6, $C$ and $D$ ). Earlier studies by Rakic $(1976 \mathrm{~b}, 1983)$ have shown that genicu-
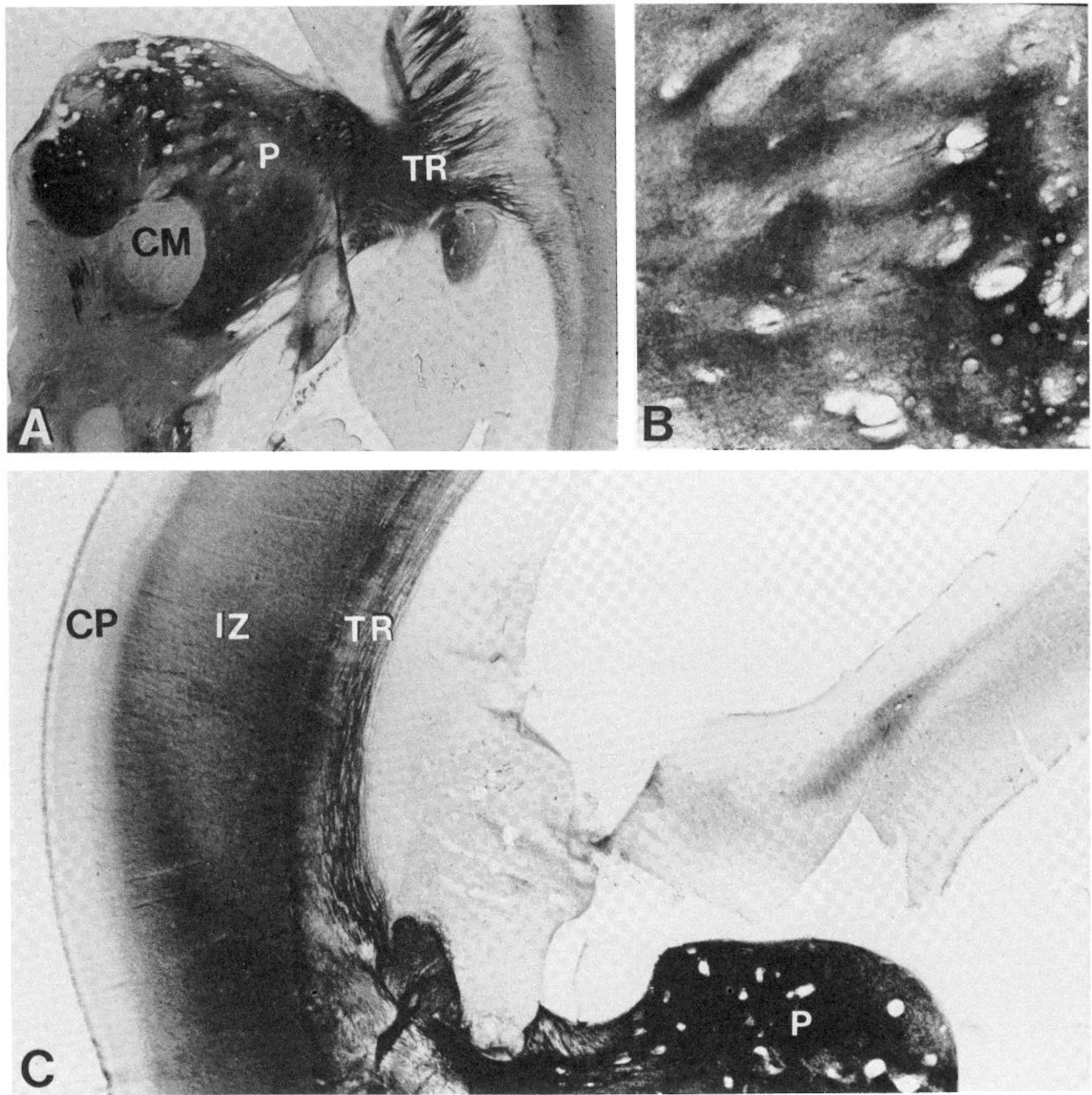

Figure 4. A, Highly positive staining of the pulvinar $(P)$ and its projections through the thalamic radiation $(T R)$ in an 18 week-old human fetus. $B$, Higher magnification of the same region displays a fenestrated staining pattern. $C$, Region of confluence between the diencephalon and cerebral vesicle showing the positively stained pulvinar $(P)$ and its fibers entering the intermediate zone $(I Z)$ of the occipitotemporoparietal territory. 

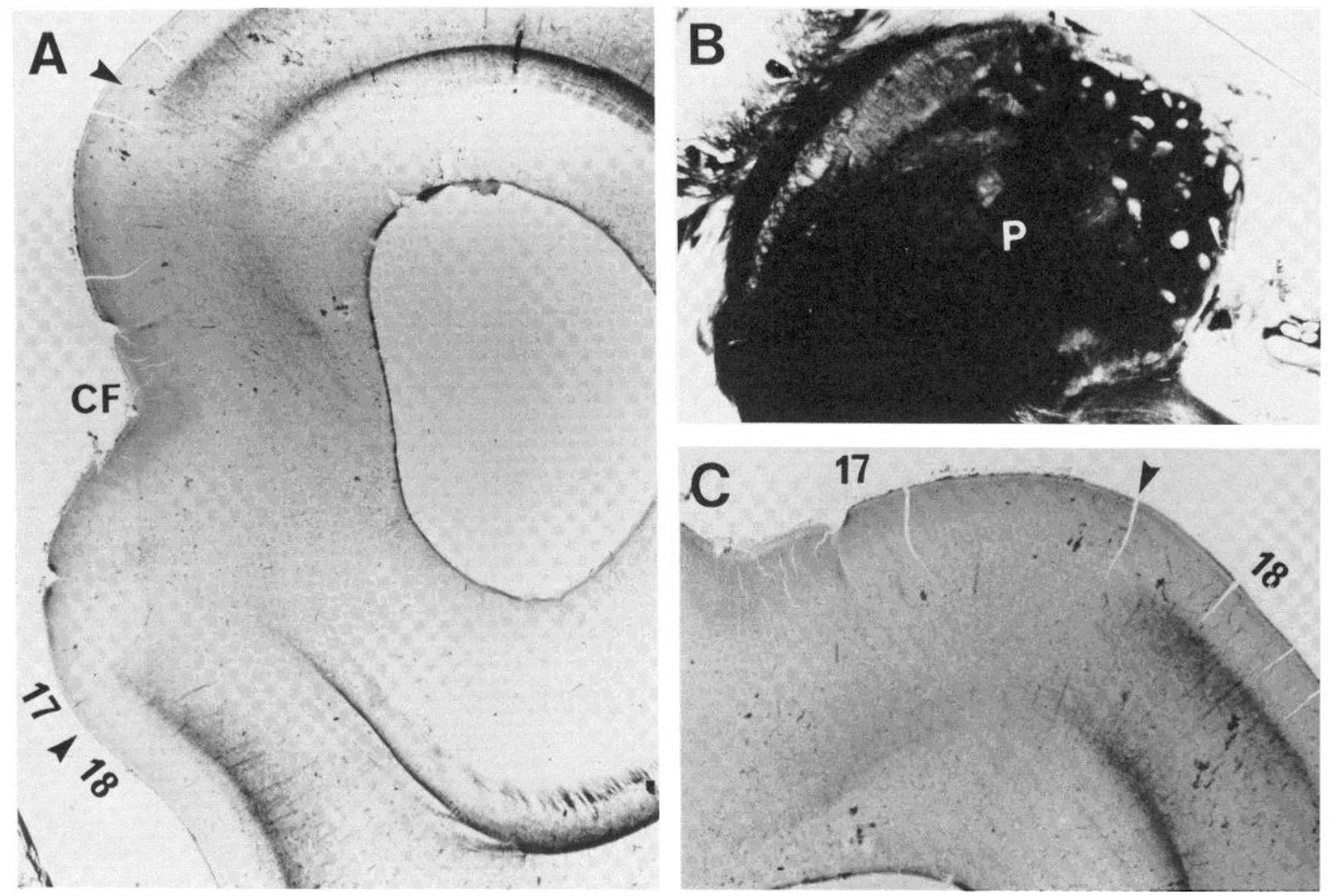

Figure 5. A, Coronal section across the occipital lobe of a 24-week-old human fetus. Many ChE-positive pulvinar axons peal off from the thalamic radiation and become distributed below the cortical plate of prospective area 18 , but do not invade the territory below future area 17 , which can be recognized by the presence of a shallow calcarine fissure $(C F)$. The area $17 / 18$ border is marked by arrowheads. $B$, Intensive ChE reactivity at its peak in the pulvinar $(P)$ of a 22 -week-old human fetus. $C$, Higher power photomicrograph of the borderline (arrowhead) between areas 17 and 18 in a 24 -week-old human fetus. Further explanation is given in the text.

locortical fibers also respect this border at the level of layer IV (Fig. $6 B$ ). In contrast, layer I and the subplate zone are slightly stained for ChE over the entire surface of the occipital lobe including the area subjacent to area 17. At the most caudal portion of the calcarine fissure, close to the tip of the occipital cortex, ChE staining of the subplate zone stops sharply at the upper and superior limb of the striate cortex. More rostrally, however, the histochemically defined border can be detected only in the inferior limb of the calcarine fissure. The lack of clear definition in the superior limb presumably reflects the overlap there between the $\mathrm{ChE}$ pulvinar projection and ChE-positive fibers of basal forebrain origin emanating from the external capsule.

Stage V. During W26 to W34 in the human and E100 to E124 in monkey fetuses, pulvinar neurons are considerably more differentiated (Ogren and Rakic, 1981). However, ChE reactivity within the nucleus is only slightly decreased and maintains a pronounced patchy distribution in both humans (Fig. 7, $A$ and $B$ ) and monkeys (Fig. 10A). Major changes do occur in the distribution and intensity of activity within the subplate zone and the cortical plate of the developing occipital lobe. The subplate zone becomes somewhat less intensively stained, although the border between the areas underlying the primary and prestriate visual cortex is still sharp. Within the prestriate cortex, staining is more intense but does not show periodicity in the form of lighter and darker patches (Figs. $8 A, 9 B$, and $10 B$ ). However, at this stage one can clearly distinguish a differential laminar distribution of ChE activity. The staining is concentrated in two darkly stained bands separated by a pale band. The most superficial strata, except layer I of the cortical plate, are unstained in both the human (Figs. 8, $A$ and $B$, and $9, A$ to $C$ ) and the monkey (Fig. 10, $B$ and $C$ ).

Because separation of the cortical plate into specific layers is still not fully established at these fetal ages (Fig. $11 C$ ), it is difficult to interpret histochemically visible lamination in terms of the layers described in the adult cortex. However, comparison of ChE-stained sections with the adjacent cresyl violet-stained sections (e.g., Fig. $11, B$ and $C$ ) suggests that the external dark band may correspond to prospective cortical layer IV and the inner half of layer III, and the deeper one may correspond to prospective layer VI. The stain-free band probably cor- 
responds to future layer $\mathrm{V}$. We should point out that the $\mathrm{ChE}$ reactivity associated with the pulvinar afferents is not fully in register with the cortical layers visible in adjacent Nissl sections because the histochemical procedure produces considerable shrinkage of the tissue and the laminar relationship becomes somewhat distorted. However, the two dark bands of positive $\mathrm{ChE}$ staining stop abruptly at the borderline between prestriate and striate cortex (Figs. 8 to 10).

In addition to the two dark bands of $\mathrm{ChE}$ reactivity,
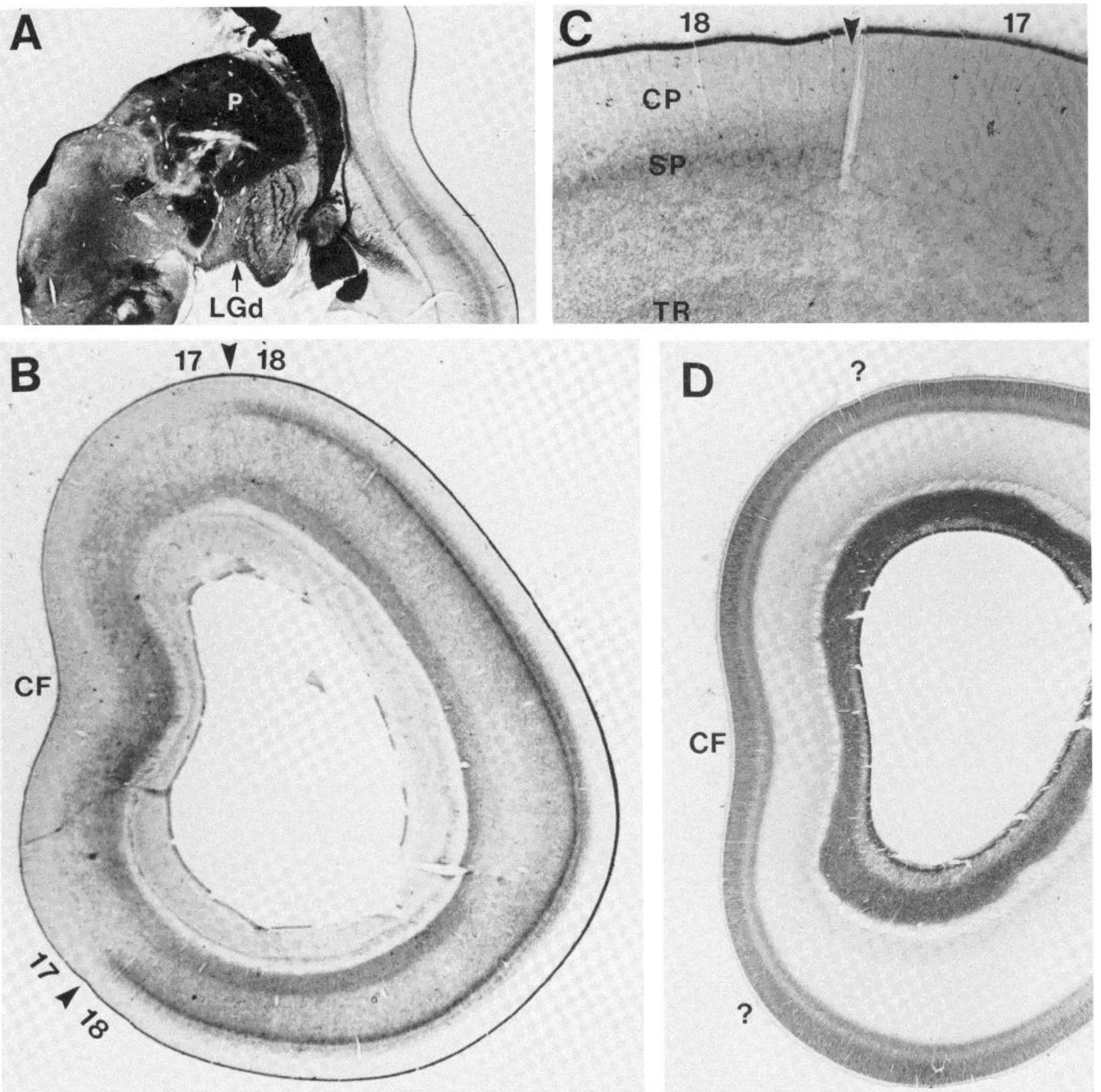

Figure 6. A, An example of positive staining of the pulvinar $(P)$ in a monkey fetus at the peak of ChE activity during stage IV. Note that the dorsal lateral geniculate nucleus $(L G d)$ remains unstained although the interlaminar spaces at this age contain ChE-positive fibers. B, Low power photomicrograph of a ChE-stained coronal section across the occipital lobe in a 93-day-old monkey fetus. Positively stained fibers are present in the subplate zone below the prospective territory of area 18 but are absent in the subplate subjacent to area 17 , which is recognized by the presence of the shallow calcarine fissure $(C F)$. $C$, Enlargement of the approximate territory of the future borderline (arrowhead) between areas 17 and 18 that is recognized at this fetal age by the presence of ChE-rich fibers in the subplate zone $(S P)$ subjacent to the cortical plate $(C P)$ of area 17 . Only a small number of fibers have entered the cortical plate at this age. $D$, Cresyl violet-stained section across the occipital lobe in the same specimen. There are no morphological signs of the cytoarchitectonic subdivisions of the occipital cortex. Question marks (?) indicate the approximate position of the prospective borderline line as determined from the adjacent ChE-stained sections. 

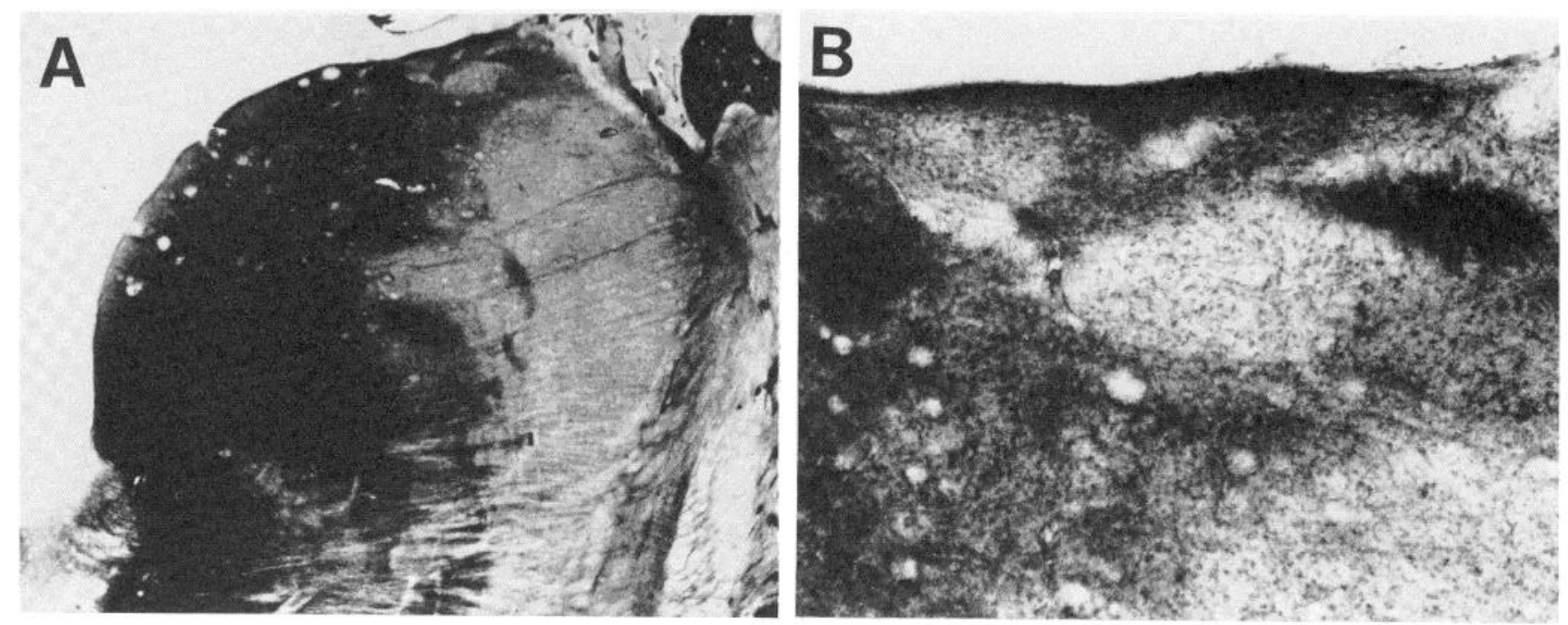

Figure 7. A, Pulvinar region in a 28-week-old human fetus stained for ChE. B, Higher power photograph of a selected field from the same section to display fenestrated distribution of the enzyme in the fourth stage.

the external portion of layer I is also stained. This band is barely visible in the human material but is clearly present in two monkey fetuses (Figs. $10 B$ and $11 B$ ). Staining in layer I does not stop at the area $17 / 18$ border; rather, it spreads over the entire territory at the occipital lobe. Diminished reactivity in layer I of the human material may reflect species-specific differences in pulvinar projections to area 17. Alternatively, the dark staining in the monkey fetuses may be a result of the better preservation of tissue by perfusion fixation.

Stage VI. ChE activity in the pulvinar gradually decreases after W35 in the human and E125 in the monkey. Although in infants some sections of the pulvinar are positively stained (Fig. 12, $A$ and $B$ ), in general, ChE activity is reduced to relatively small, lightly stained patches scattered within the neuropil. By the end of the 6 th month in humans and the 1st postnatal month in monkeys, the pulvinar is histochemically distinguished from the adjacent thalamic nuclei although some faintly ChE-positive neurons can be observed. However, axons of such cells are not stained well enough to be traced to the occipital lobe. Although in the mature human and monkey brain some cells and surrounding neuropil may show a minimal amount of reactivity (see also Manocha and Shantha, 1970), most pulvinar neurons are unstained. The direct coloring method gives the impression of a more positive reaction, particularly when intensified with silver nitrate (see Olivier et al., 1970; GeneserJensen and Blackstad, 1971; Graybiel and Berson, 1980). However, in our material stained using a modification of the acetylthiocholine iodide method, axons emerging from the pulvinar are unstained and the prestriate cortex of both human and monkey brain shows a significant decrease in staining intensity throughout all cortical layers.

In contrast to the decline in $\mathrm{ChE}$ activity in the prestriate cortex, area 17 begins to display a rich $\mathrm{ChE}$ positive axonal network (Fig. 12B). Owing to the emergence of $\mathrm{ChE}$ positivity in striate cortex, the distinction between areas 17 and 18 is much less well defined in adults. In adult brain, area 17 contains increased num- bers of ChE-positive fibers, particularly in layers I, IVA, and VI as shown by Graybiel and Ragsdale (1982) and Hedreen et al. (1982). These changes in the cortex occur in parallel with the decrease in $\mathrm{ChE}$ in the pulvinar (Fig. $12 \mathrm{~A}$ ) and enhancement of reactivity in the laminae of the LGd (Figs. $12 C$ and $13 B$ ). In both the monkey and human we found slightly higher $\mathrm{ChE}$ reactivity in the magnocellular than in parvocellular layers (Fig. 13B), in agreement with Dean et al. (1982). It should be emphasized, however, that the ChE staining of the LGd laminae is probably due mainly to the reactivity of various afferent fiber systems as experimentally demonstrated in the adult brains of several other primate species such as the bushbaby and the owl monkey (Fitzpatrick and Diamond, 1979, 1980).

\section{Discussion}

The role and the significance of transient $\mathrm{ChE}$ activity in selected populations of neurons in the developing brain are unknown. There is some indication that $\mathrm{AChE}$ in embryos has a function and several molecular forms which differ from those in the adult (Davis and Agranoff, 1968; Silver, 1974; Vijayan and Oschowska, 1977; Wade and Timiras, 1980). The widespread presence of these molecules throughout the animal kingdom, even in organs and organisms that lack neural tissue such as spermatozoa (Sekine, 1951), protozoa (Seaman and Houlihan, 1951), or echinoidea (Gustafson and Toneby, 1970), indicates that various forms of ChE may have a developmental function unrelated to neuronal transmission. Although none of the hypotheses about the nontransmitter role of AChE have been substantiated so far (Buznikov, 1980), the presence of this molecule at early phylogenetic and ontogenetic stages suggests the possibility of a morphogenetic function. In spite of our almost total ignorance about the role and significance of $\mathrm{ChE}$ during development, its presence nevertheless can be used to determine the time course and pattern of development of well defined neuronal populations or pathways. For example, during mid-gestation when other anatomical methods are not feasible, the ChE method 
may be used to examine and compare the development of pulvinar projections to the neocortex in humans and monkeys. Conveniently, the pulvinar and its projections can be identified because the nearby thalamic nuclei which project to adjacent cortical areas are mostly ChE negative during development.

Developmental schedule of projections to prestriate cortex. The development time course for extrageniculate visual projections to the occipital lobe is determined in this study on the basis of their stainability with the acetylthiocholine iodide method. Obviously, if some axons originating from the pulvinar were present earlier but did not contain stainable substance, they would be overlooked. However, there are several sources of evidence indicating that $\mathrm{ChE}$ reactivity is present in some actively growing axonal processes and that this histochemical method, at least in the case of certain thalamocortical connections, can be used to determine the time and sequence of their development relatively accurately. First, the ingrowth of pulvinar fibers follows relatively closely the ingrowth of adjacent geniculocortical fibers labeled by anterograde transneuronal transport of radioactive tracers injected into one eye (Rakic, 1976b, 1983). Second, because both LGd and pulvinar
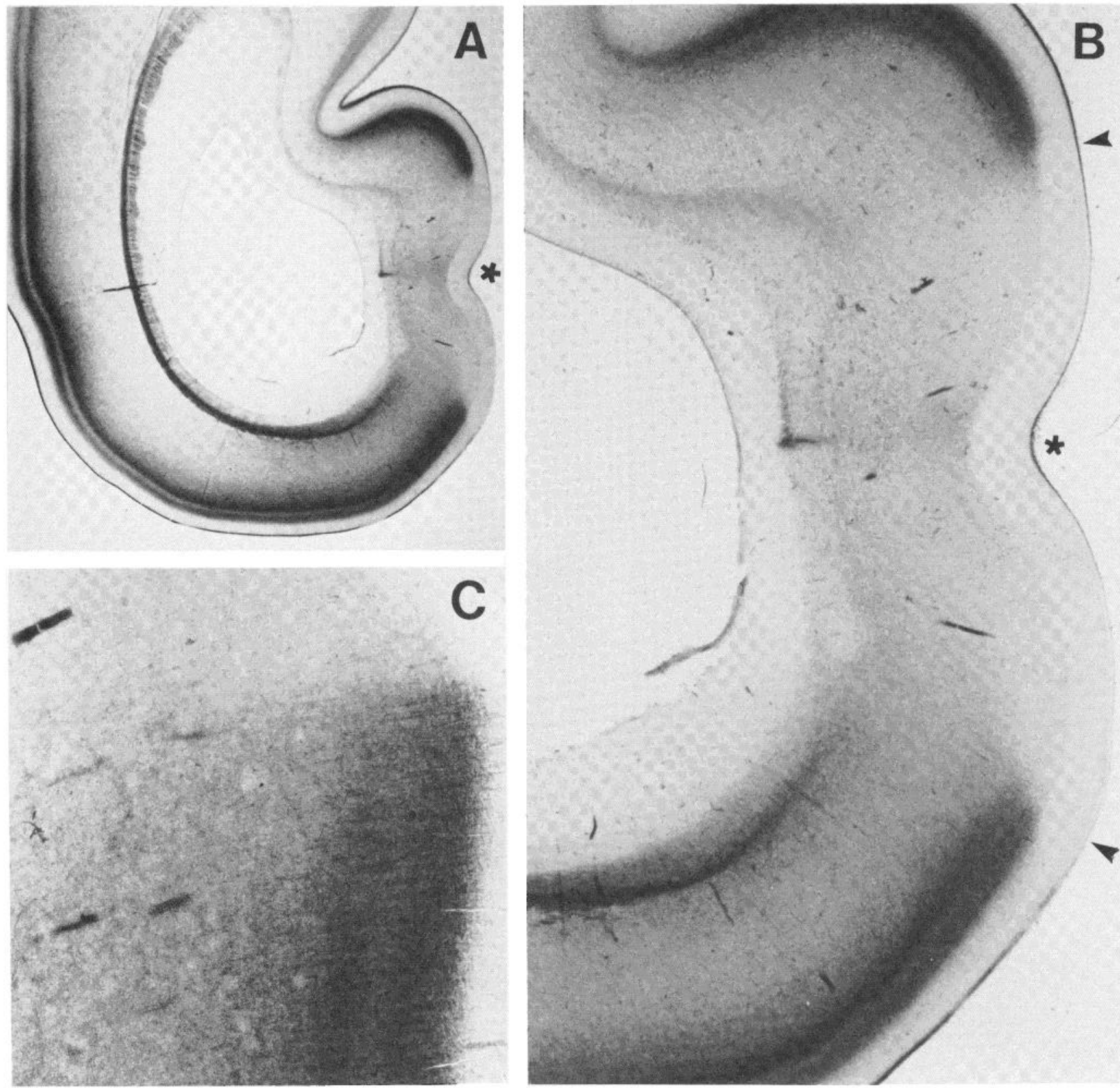

Figure 8. A, Section across the occipital lobe in the 26-week-old human fetus stained using the ChE method. By this fetal age, positively stained fibers are present in the cortical plate itself. The calcarine fissure is indicated by an asterisk. B, Enlargement of the area around the calcarine fissure $\left({ }^{*}\right)$ illustrates the rather abrupt falloff in ChE staining in the cortex at the borderline between prospective areas 17 and 18 (arrowheads). $C$, Higher magnification of the borderline territory between stained and unstained cortex in the same specimen. Note a faint bilaminar distribution of ChE reactivity. 
neurons are generated at essentially the same time (Rakic, 1977a) and their neurons appear to mature at corresponding ages (Ogren and Rakic, 1981), it would be expected that they would also develop their axons at approximately the same time. Third, as soon as the initial fiber fascicles appear as unstained bundles exiting from the anlage of the pulvinar in the Nissl-stained sections, they are $\mathrm{ChE}$ positive in adjacent histochemically reacted sections. Finally, other developing neuronal systems are also stained most intensively at the stages when major axonal connections leave and/or invade a given structure (Krnjevic and Silver, 1966; Kostovic and Goldman-
Rakic, 1983). Nevertheless, we cannot exclude the possibility that a small number of pioneer axons originate from the pulvinar and enter the occipital lobe or even invade the cortical plate at stages earlier than reported in the present study. It should be pointed out that this is a possibility that cannot be excluded in any developmental study that utilizes currently available morphological methods including monoamine histochemistry or retrograde and anterograde axonal transport methods. Yet, these tracing methods as well as ChE histochemistry provide an important, and currently, the only available approach to study relative sequences, timing, and pat-
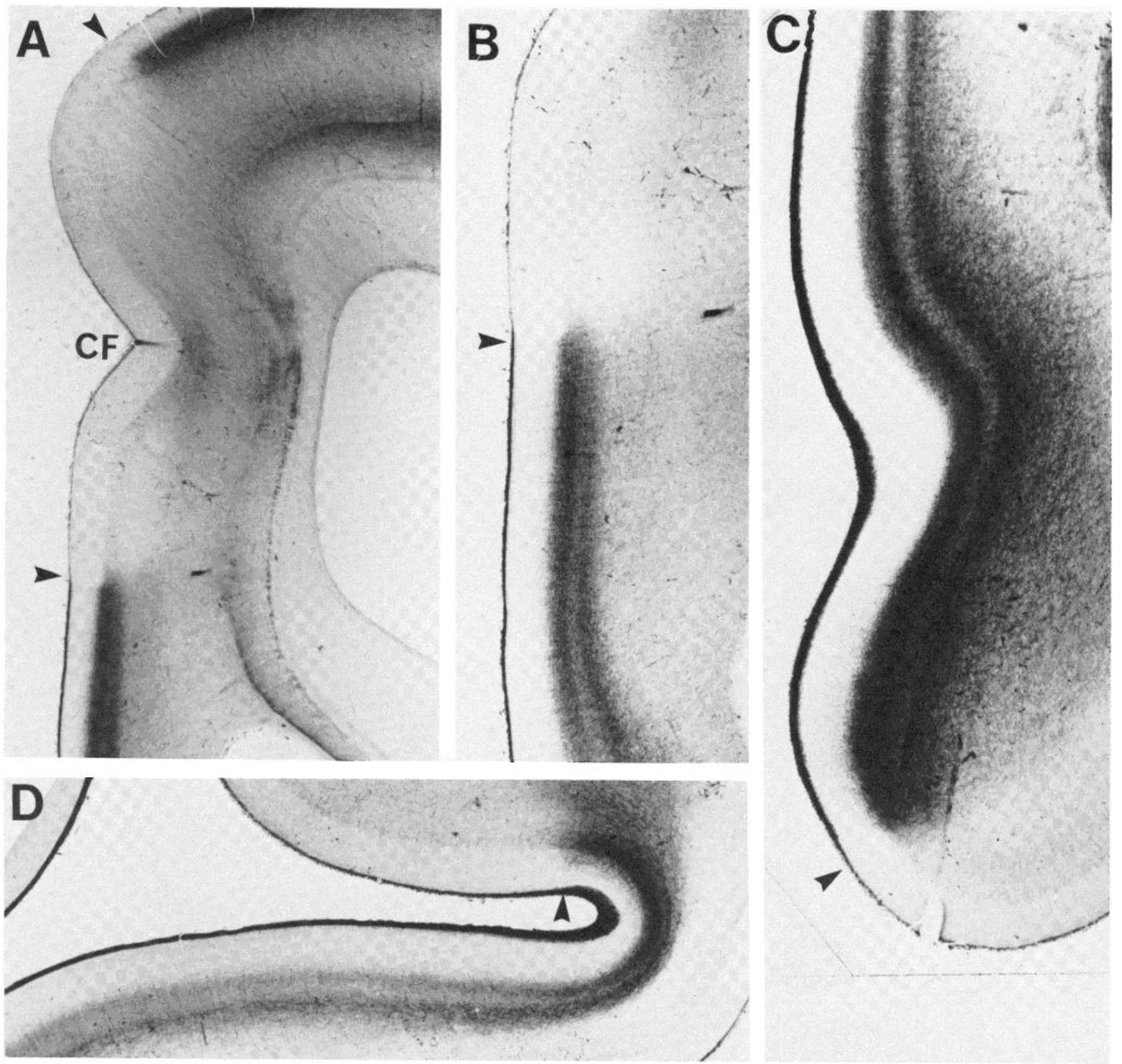

Figure 9. A, An example of the abrupt change in ChE reactivity at the border between area 18 and prospective area 17 in the depth of the calcarine fissure $(C F)$ in a 26-week-old human fetus. $B$, Higher power photographs of the border zone between stained and unstained territories in the lower bank of the calcarine fissure, which displays a faint bilaminar distribution of pulvinar axons. $C$, Upper bank of the calcarine fissure with border of stained and unstained territories. $D$, Another example of the area $17 / 18$ border indicated by the arrowhead. 

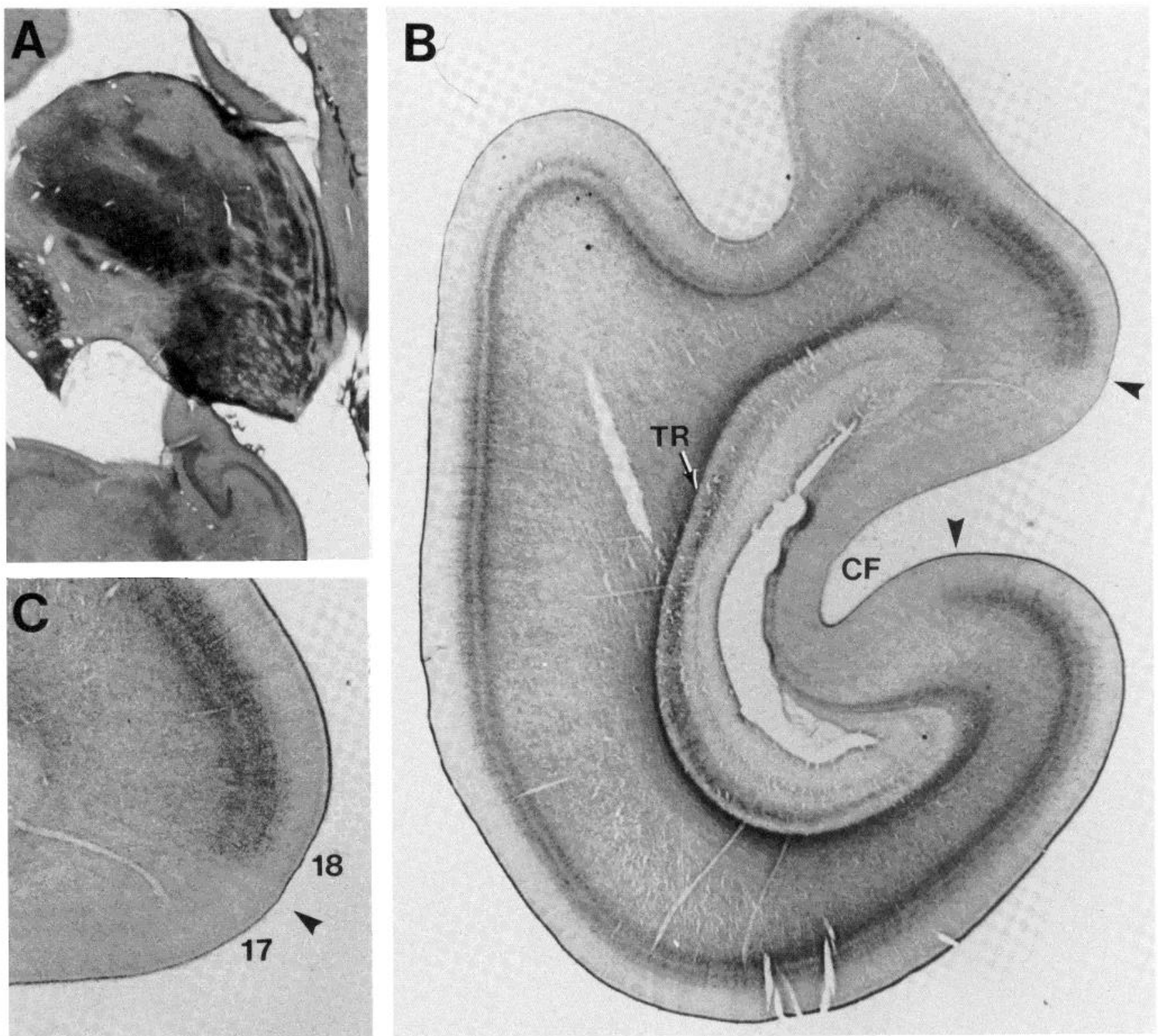

Figure 10. A, Posterior thalamus stained for ChE in an E123 monkey fetus to illustrate decline in staining intensity in the pulvinar. $B$, Coronal section across the occipital lobe in an E102 monkey fetus. Thalamic radiation $(T R)$ is densely stained and many ChE-positive fibers have entered the cortex and are distributed in a bilaminar fashion. The territory in the depth of the calcarine fissure $(C F)$ remains stained. $C$, Higher power photomicrograph of the border (arrowhead) between areas 17 and 18 in the upper lip of the calcarine fissure.

terns of fiber ingrowth to a given cortical area in mammals.

Comparison of the onset, tempo, and sequences of development of prestriate afferents in the two primate species show remarkable similarity. However, each individual developmental stage in the monkey is somewhat shorter and occurs slightly earlier in relation to birth than it does in the human. As a consequence, the newborn monkey possesses a slightly more advanced pattern of connectivity of the prestriate visual cortex than does the newborn human. Nevertheless, in comparison to the tempo of neurogenesis of the visual system in rodents and carnivores (Anker and Cragg, 1974; LeVay et al., 1978) the entire visual system in both primate species examined in the present study reaches a considerably higher level of differentiation during intrauterine life.

Mode of pulvinar projections to developing prestriate cortex. Observations made on $\mathrm{ChE}$ histochemical material indicate that development of thalamic projections to the prestriate cortex follow basically the same developmental sequences and rules that were described for the genesis of the geniculocortical axonal system that projects to the striate cortical area (Rakic, 1976b, 1983) and, more recently, for the mediodorsal projection to the frontal cortex (Kostovic and Goldman-Rakic, 1983). In the first phase pulvinar fibers enter the intermediate zone of the occipital lobe but do not invade the developing cortical plate which still lacks a majority of its 
neurons. In the next phase, pulvinar fibers accumulate within the subplate zone but their concentration is particularly high in the sectors directly below the prospective prestriate cortex of the occipitotemporoparietal confluence. It is likely that the accumulation of $\mathrm{ChE}$ fibers in the subplate zone is enhanced by a contribution from the external capsule radiation which also stains intensely early in both monkey and human development (Kostovic and Goldman-Rakic, 1983). However, the ChE-positive areas in the subplate zone is clearly delineated from the adjacent territories that do not contain reaction product and presumably do not possess a large contingent of pulvinar or basal forebrain axons. The unstained area is situated below the cortical plate at the occipital lobe where one can expect development of striate cortex. The border between the stained and unstained areas in the subplate zone is initially diffuse but gradually becomes more sharply defined. As in the case of geniculocortical fibers (Rakic, 1976a, 1977b), the subplate zone may be considered a "waiting compartment" for afferent axons. The thalamic fibers may be stored in this zone until all or most of their target neurons are generated and have reached their positions within the cortex.

In the final stage, pulvinar fibers enter the developing cortical plate. However, from the very start, they seem to avoid prospective striate cortex and exclusively invade the prestriate areas that eventually have massive pulvinar projections. Except in layer I, ChE reactivity stops sharply at the presumed area $17 / 18$ border. This abrupt change of staining property is localized in the portions of the calcarine fissure that roughly correspond to the area 17/18 border in older cases where cytoarchitectonic differences emerge. Furthermore, the ChE staining stops at the point where geniculocalcarine fibers enter the striate cortex as visualized by transneuronal transport of radioactive tracers after intraocular injections in fetal
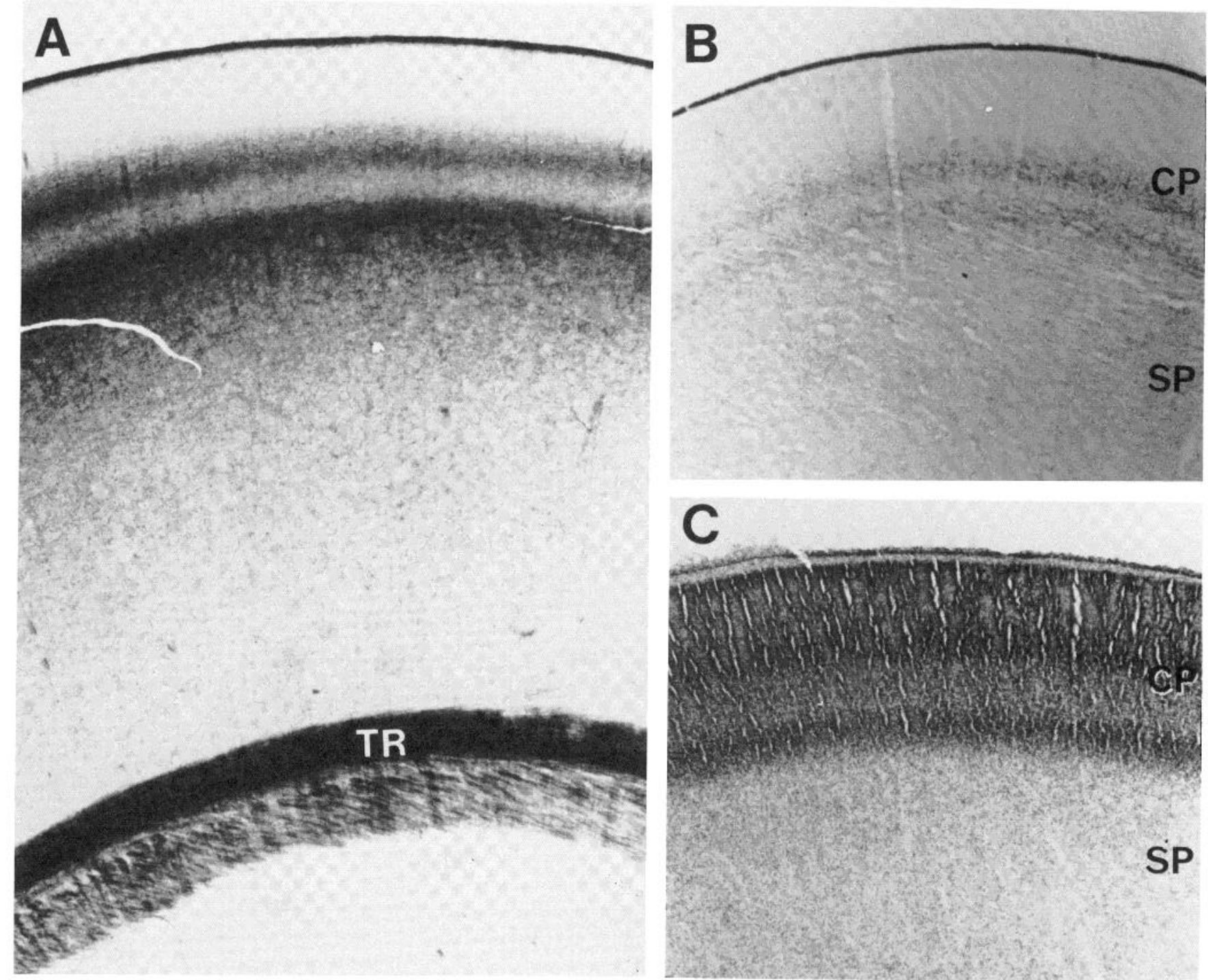

Figure 11. A, Prestriate area in a 26 -week-old human fetus. Intensively stained fibers peal off from the thalamic radiation $(T R)$ and become distributed within the subplate and cortical plate in a bilaminar fashion. $B$, Similar bilaminar distribution of $\mathrm{ChE}$ reactivity in the cortical plate $(C P)$ of an E123 monkey fetus with a smaller amount of activity dispersed throughout the subplate zone $(S P)$. C , Cresyl violet-stained section adjacent to the one shown in $B$. Although the subplate $(S P)$ and cortical plate $(C P)$ are easily recognized, segregation into horizontal lamination only has begun at this fetal age. 

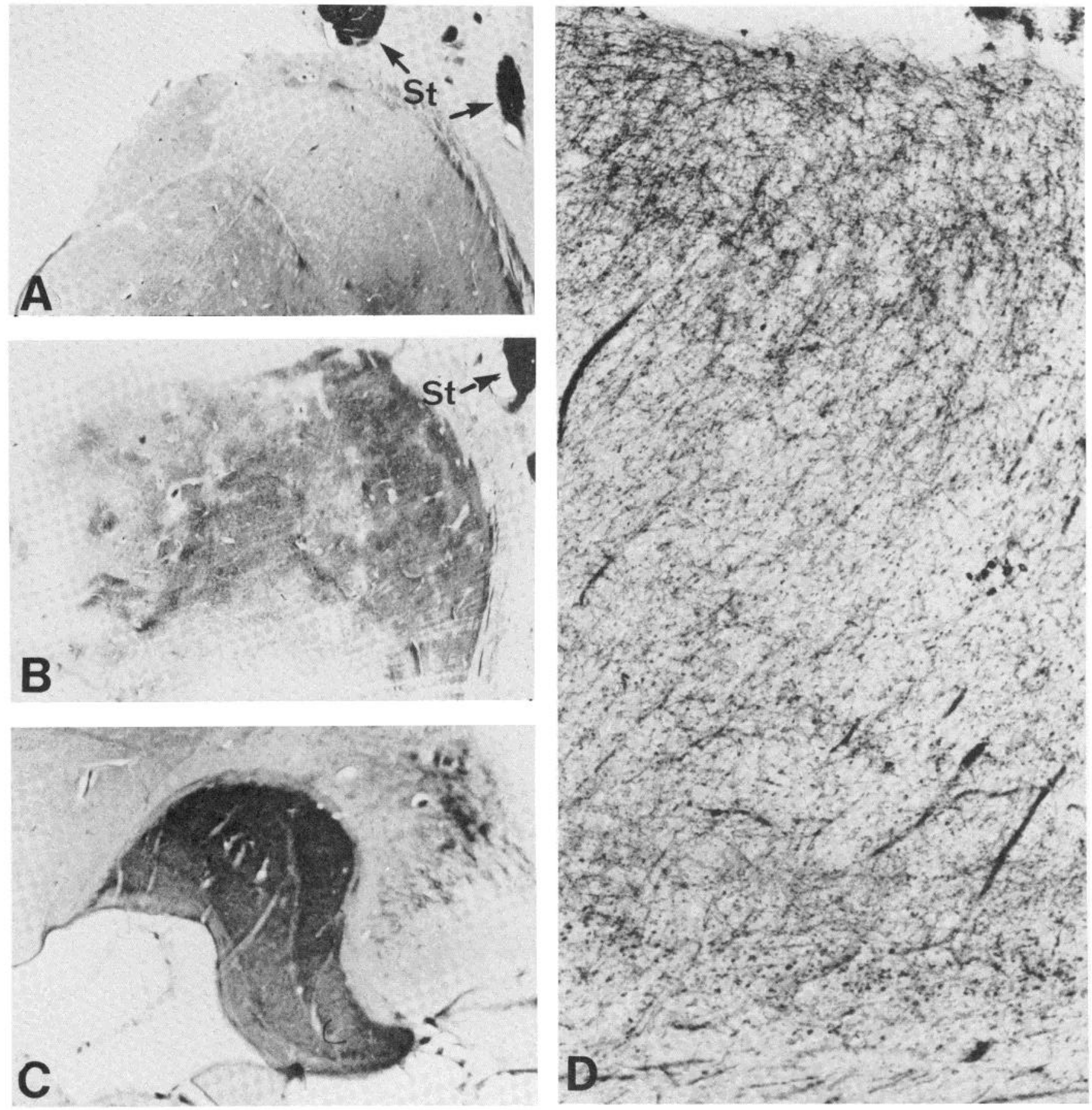

Figure 12. A, The pulvinar in a 3-month-old human infant shows pale ChE reactivity while the posterior thalamic radiation which contains axons from the pulvinar is unstained. In contrast, the body of the caudate nucleus (arrows) is intensely stained. $B$, Section through the more posterior portion of the pulvinar in the same case shown in $A$. ChE reactivity is low and patchy compared to intensive staining of the adjacent lateral geniculate nucleus (see $C$ ) or the caudate that is present in the same section (Fig. 12A). C, ChE staining of the lateral geniculate nucleus in the same infant. Note moderately strong reactivity in the cellular layers and stain-free interlaminar spaces. $D$, Striate area in a 3-month-old human infant. Note the differential distribution of the fine network of ChE-positive fibers (see "Discussion").

monkeys (Rakic, 1976b, 1977b, 1983). Although a small contingent of pulvinar neurons also projects to the striate cortex in the rhesus monkey (Ogren and Hendrickson, 1977; Rezak and Benevento, 1979; Lund et al., 1981), the distinctive laminar distributions in the two areas are clear. During development pulvinar projections to the striate cortex are barely visible and terminate mostly in the superficial portions of layers I and II, avoiding other layers of the cortex, whereas pulvinar projection to the prestriate cortex is more voluminous and terminates mostly in two deep lying bands in this region.

At developmental stages when pulvinar cortical input 

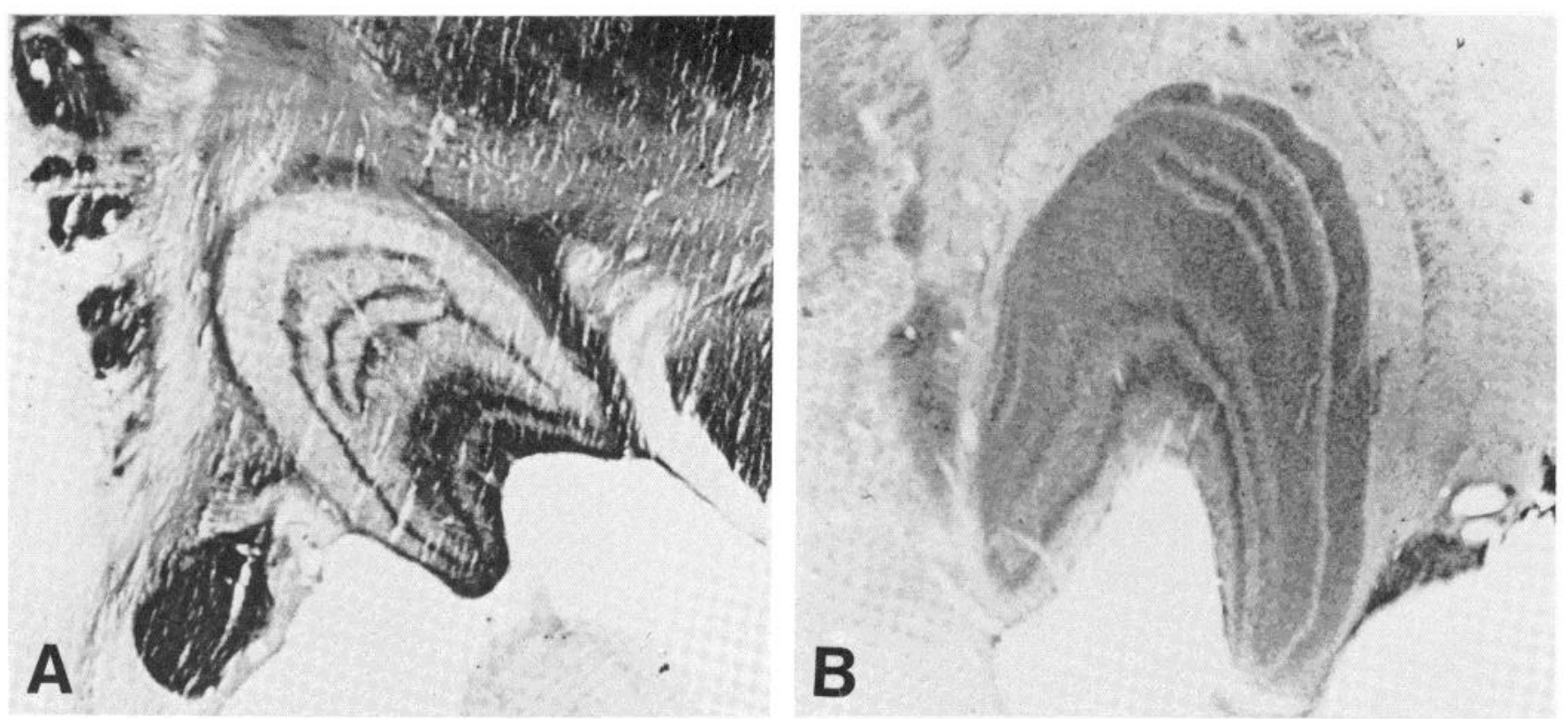

Figure 13. A, ChE-positive staining of the intralaminar spaces in the lateral geniculate nucleus in an E102 monkey fetus. $B$, Lateral geniculate nucleus in an older fetal monkey (E123) in whom intralaminar spaces have lost ChE reactivity while the cellular laminae became moderately stained.

can be stained with the ChE method, it displays clear laminar differentiation. However, we have not seen horizontal periodicity in the form of alternating stripes that are characteristic of this afferent system in adult monkeys (Ogren and Hendrickson, 1977; Curcio and Harting, 1978). One can presume that the segregation into stripes may occur at later stages in a manner similar to the segregation of ocular dominance stripes in the striate area (Rakic, 1976b; Hubel et al., 1977). However, the processes of sorting out of the pulvinar input cannot be revealed by the present method since it occurs at the later stages when the transient $\mathrm{ChE}$ reactivity of the pulvinar axons disappears. The precise and clearly targeted development of projections observed for both striate (Rakic, 1976a, 1983) and extrastriate afferents suggests that thalamic axons might recognize their targets before any visible sign of cytoarchitectonic differentiation within the cortical plate. Conversely, one can argue that pulvinar axons grow to the "appropriate" cortical area because the adjacent cortex is populated by fibers that have arrived earlier or simultaneously from the adjacent thalamic LGd nucleus. One can speculate even further that the border between striate and prestriate areas may actually be created by the competition between geniculate and pulvinar axons for cortical neurons on the border and that this axon-axon competition indirectly determines the limits of boundaries between cytoarchitectonic parcels (e.g., see Goldman-Rakic and Rakic, 1984). This hypothesis seems to be complicated by the finding that a small number of pulvinar axons do enter the striate area, although these appear to seek different synaptic targets that are situated mostly in layer I and II, rather than in the deeper layers, particularly IV, III, and VI, which are preferentially innervated in the prestriate area. Likewise, a small number of LGd fibers invade prestriate areas (Yukie and Iwai, 1981) and terminate mostly in layer V (Benevento and Yoshida, 1981); they avoid layers I, III, and VI, the preferred targets of pulvinar axons in this cytoarchitectonic field. The differential distribution of thalamic fibers between the striate and prestriate cortex described in the present study occurs initially before the main cytoarchitectonic boundaries of the occipital lobe become visible in Nisslstained sections. At present we cannot distinguish between the effects of axonal competition and the possibility that the cortical plate may contain a heterogeneous population of neurons that, although morphologically similar, already possess surface properties that are recognizable by the ingrowing fibers (Rakic, 1981, 1983; Rakic and Goldman-Rakic, 1982). These important developmental issues remain unresolved. However, our findings suggest that the border between striate and prestriate cortex may be an excellent model for experimental studies of the development of cytoarchitectonic parcellations in the primate cerebrum.

\section{References}

Anker, R. L., and B. G. Cragg (1974) Development of the extrinsic connections of the visual cortex in the cat. J. Comp. Neurol. 154: 29-42.

Benevento, L. A., and M. Rezak (1976) The cortical projections of the inferior pulvinar and adjacent lateral pulvinar in the rhesus monkey (Macaca mulatta): An autoradiographic study. Brain Res. 108: 1-24.

Benevento, L. A., and K. Yoshida (1981) The afferent and efferent organization of the lateral geniculo-prestriate pathways in the macaque monkey. J. Comp. Neurol. 203: 455474 .

Broderson, S. H., L. E. Westrum, and A. E. Sutton (1974) Studies of the direct coloring thiocholine method for localizing cholinesterase activity. Histochemistry 40: 13-23.

Buznikov, G. A. (1980) Biogenic monoamines and acetylcholine in protozoa and metazoan embryos. In Neurotransmitters, 
Comparative Aspects, J. Salanki and T. M. Turpaev, eds., pp. 7-29, Academiai Kiado, Budapest.

Cragg, B. G. (1969) The topography of the afferent projections in the circumstriate visual cortex of the monkey studied by the Nauta method. Vision Res. 9: 733-747.

Csillik, B., F. Joo, P. Dasa, I. Yomity, and G. Kalman (1964) Development of acetyocholinesterase-active structures in the rat archicerebellar cortex. Acta Biol. Hung. 15: 11-17.

Curcio, C. A., and J. K. Harting (1978) Organization of pulvinar afferents to area 18 in the squirrel monkey: Evidence for stripes. Brain Res. 174: 155-161.

Davis, G. A., and B. W. Agranoff (1968) Metabolic behavior of isoenzymes of acetylcholinesterase. Nature 220: 227-280.

Dean, A. F., S. T. Bunch, D. J. Tolhurst, and P. R. Lewis (1982) The distribution of acetylcholinesterase in the lateral geniculate nucleus of the cat and monkey. Brain Res. 244: 123134.

Filogamo, G., and P. C. Marchisio (1965) Acetylcholine system and neural development. Neurosci. Res. 4: 29-46.

Fitzpatrick, D., and J. T. Diamond (1979) The laminar organization of the lateral geniculate body in Galago senegalensis: A pair of layers identified by acetylcholinesterase activity. Brain Res. 170: 538-542.

Fitzpatrick, D., and J. T. Diamond (1980) Distribution of acetylcholinesterase in the genicula striate system of Galago senegalensis and Aotes trivirgatus: Evidence for origin of the reaction products in the lateral geniculate body. J. Comp. Neurol. 194: 703-719.

Geneser-Jensen, F. A., and J. W. Blackstad (1971) Distribution of acetylcholinesterase in the hippocampal region of the guinea pig. I. Entorhinal area,- parasubiculum and presubiculum. Z. Zellforsch. Mikrosk. Anat. 114: 460481.

Goldman-Rakic, P. S., and P. Rakic (1983) Experimentally modified convolutional patterns in nonhuman primates: Possible relevance of connections to cerebral dominance in man. In Biological Foundations of Cerebral Dominance, N. Geschwind, ed., Harvard University Press, Cambridge, MA, in press.

Graybiel, A. M., and D. M. Berson (1980) Histochemical localization and afferent connections of subdivisions in the lateralis posterior-pulvinar complex and related thalamic nuclei in the cat. Neuroscience 5: 12-38.

Graybiel, A. M., and C. W. Ragsdale (1982) Pseudocholinesterase staining in the primary visual pathways of the macaque monkey. Nature 299: 439-442.

Gustafson, T., and M. Toneby (1970) On the role of serotonin and acetylcholine in sea urchin morphogenesis. Fxp. Cell Res. 62: 102-117.

Hedreen, J. C., A. R. Uhl, S. J. Uhl, C. L. White, D. L. Price, and D. M. Fambrough (1982) A fiber network in monkey cerebral cortex revealed by acetylcholinesterase immunocytochemistry. Soc. Neurosci. Abstr. 8: 212.

Hubel, D. H., T. N. Wiesel, and S. LeVay (1977) Plasticity of ocular dominance in monkey striate cortex. Philos. Trans. R. Soc. Lond. (Biol.) 278: 377-409.

Karnovsky, M. J., and L. Roots (1964) A "direct coloring" thiocholine method for cholinesterase. J. Histochem. Cytochem. 12: 219-221.

Kostovic, I., and P. S. Goldman-Rakic (1983) Transient acetylcholinesterase activity in the mediodorsal nucleus of the thalamus and its connections in the developing human and monkey brain. J. Comp. Neurol. 219: 431-447.

Kostovic, I., and M. E. Molliver (1974) A new interpretation of the laminar development of cerebral cortex: Synaptogenesis in different layers of neopallum in the human fetus. Anat. Rec. 178: 395.

Krnjevic, K., and A. Silver (1965) A histochemical study of cholinergic fibers in the cerebral cortex. J. Anat. 99: 711759 .
Krnjevic, K., and A. Silver (1966) Acetylcholinesterase in the developing forebrain. J. Anat. 100: 63-89.

LeVay, S., M. P. Stryker, and C. J. Shatz (1978) Ocular dominance columns and their development in layer IV of the cat's visual cortex: A quantitative study. J. Comp. Neurol., 179. $223-244$.

Lund, J. S., A. E. Hendrickson, M. P. Ogren, and A. Tobin (1981) Anatomical organization of primate visual cortex area VII. J. Comp. Neurol. 202: 19-45.

Manocha, S. L., and T. R. Shantha (1970) Macaca Mulatta: Enzyme Histochemistry of the Nervous System, Academic Press, Inc., New York.

Ogren, M. P., and A. E. Hendrickson (1977) The distribution of pulvinar terminals in visual areas 17 and 18 of the monkey. Brain Res. 137: 334-350.

Ogren, M. P., and P. Rakic (1981) The prenatal development of the pulvinar in the monkey. ${ }^{3} \mathrm{H}$-thymidine autoradiographic and morphometric analyses. Anat. Embryol. 162: 120.

Olivier, G., and H. Pineau (1961) Horizons de Streeter et áge embryonnaire. Bull. Assoc. Anat. (Nancy) 47e: 573-576.

Olivier, A., A. Parent, and L. J. Porier (1970) Identification of the thalamic nuclei on the basis of their cholinesterase content in the monkey. J. Anat. 106: 37-50.

Rakic, P. (1972) Mode of cell migration to the superficial layers of fetal monkey neocortex. J. Comp. Neurol. 145: 61-83.

Rakic, P. (1974a) Embryonic development of the pulvinar-LP complex in man. In The Pulvinar-LP Complex, I. S. Cooper, M. Riklan, and P. T. Rakic, eds., pp. 3-25, Charles C Thomas, Springfield, IL

Rakic, P. (1974b) Neurons in rhesus monkey visual cortex: Systematic relation between time of origin and eventual disposition. Science 183: 425-427.

Rakic, P. (1976a) Differences in the time of origin and in eventual distribution of neurons in areas 17 and 18 of visual cortex in rhesus monkey. Exp. Brain Res. Suppl. 1: 244-248.

Rakic, P. (1976b) Prenatal genesis of connections subserving ocular dominance in the rhesus monkey. Nature 261: 467471.

Rakic, P. (1977a) Genesis of the dorsal lateral geniculate nucleus in the rhesus monkey: Site and time of origin, kinetics of proliferation, routes of migration and pattern of distribution of neurons. J. Comp. Neurol. 176: 23-52.

Rakic, P. (1977b) Prenatal development of the visual system in the rhesus monkey. Philos. Trans. R. Soc. Lond. (Biol.) 278: $245-260$.

Rakic, P. (1981) Developmental events leading to laminar and areal organization of the neocortex. In The Cerebral Cortex, F. D. Schmitt, ed., pp. 7-28, MIT Press, Cambridge, MA.

Rakic, P. (1983) Geniculo-cortical connections in primates: Normal and experimentally altered development. Prog. Brain Res. 58: 393-404.

Rakic, P., and P. S. Goldman-Rakic (1982) Development and modifiability of the cerebral cortex. Neurosci. Res. Program Bull. 20: 429-611.

Rakic, P., and R. L. Sidman (1969) Telencephalic origin of pulvinar neurons in the fetal human brain. Z. Anat. Entwicklungsgesch. 129: 53-82.

Rezak, M., and L. A. Benevento (1979) A comparison of the organization of the projections of the dorsal lateral geniculate nucleus, the inferior pulvinar and adjacent lateral pulvinar to primary visual cortex (area 17) in the macaque monkey. Brain Res. 167: 19-40.

Seaman, G. R., and R. K. Houlihan (1951) Enzymatic systems in Tetrahymena geleii $\mathrm{S}$. II. Acetylcholinesterase activity. Its relation to motility of the organism and to coordinated ciliary action in general. J. Cell. Comp. Physiol. 37: 309-321.

Sekine, T. (1951) Choline esterase in pig spermatozoa. J. Biochem. (Tokyo) 38: 171-179. 
Sidman, R. L., and P. Rakic (1973) Neuronal migration, with special reference to developing human brain: A review. Brain Res. 62: 1-35.

Silver, A. (1974) The Biology of Cholinesterases, Elsevier-North Holland Publishing Co., Amsterdam.

Vijayan, V. K., and J. A. Oschowska (1977) Brain acetylcholinesterase activity and multiplicity in Bonnet monkey, Macaca radiata, and the rhesus monkey (Macaca mulatta). J. Neurochem. 28: 1141-1143.

Wade, P. D., and P. S. Timiras (1980) A regional study of the molecular forms of acetylcholinesterase in the brain of developing and adult rats. Dev. Neurosci. 3: 101-108.
Yakovlev, P. I. (1969) The development of the nuclei of the dorsal thalamus and of the cerebral cortex: Morphogenetic and tectogenetic correlation. In Modern Neurology: Papers in Tribute to Derek Denny-Brown, S. Locke, ed., pp. 15-53, Little, Brown and Co.

Yukie, M., and E. Iwai (1981) Direct projection from the dorsal lateral geniculate nucleus to the prestriate cortex in macaque monkeys. J. Comp. Neurol. 201: 81-97.

7eki, S. M. (1969a) The secondary visual areas of the monkey. Brain Res. 13: 197-226.

Zeki, S. M. (1969b) Representation of central visual fields in prestriate cortex of monkey. Brain Res. 14: 271-291. 